\title{
COMPUTATIONAL SOLUTION OF TWO-POINT BOUNDARY VALUE PROBLEMS WITH DERIVATIVE BOUNDARY CONDITIONS USING CUBIC-SPLINE PROCESS
}

\author{
N. AMAR NATH ${ }^{1}$, DR. K. SHARATH BABU ${ }^{2}$ \& DR. R.KEDARNATH ${ }^{3}$ \\ ${ }^{I}$ Department of Science \& Humanities, MLR Institute of Technology, Hyderabad, India \\ ${ }^{2}$ Faculty of Mathematics, Matrusri Engineering College, Hyderabad, India
}

${ }^{3}$ Delivery Manager, Release Point, Hyderabad, India

\begin{abstract}
An attempt has been made here to study a two -point BVP with the mixed BCs. A cubic spline function is used to arrive at the solution of the second-order linear two-point BVPS with considerable slope conditons. While implementing the method we are applied three point recursion algorithm termed as "Thomas algorithm ", after incorporating the cubicsplines involves some parameters. These parameters may be suitably chosen to obtain an non-oscillation solution of the governing equation. The same method has been implemented on some linear problems and realised, the numerically computed results are in line with the analytical solutions available in the literature.

KEYWORDS: Boundary Value Problems (BVPs), Boundary Conditions (BCs) Cubic Spline (CS), Thomas Algorithm, Differential Equation (DE).
\end{abstract}

Received: Jun 08, 2020; Accepted: Jun 28, 2020; Published: Aug 18, 2020; Paper Id.: IJMPERDJUN2020784

\section{INTRODUCTION}

Two point BVPs are common in numerous fields of Engineering and more so in study of Fluids, Convection dominated flow problems with high Reynolds number, singularly perturbed problems, Hydrodynamics etc. Such kind of problems cannot be solved analytically always due to the methods available in the literature. Recourse must be adopted to get the nature of such system by applying parallel computing, numerical methods. Particularly in the numerical methods we come across FEM, FDM and FVM. In these methods we have to develop a grid or mesh in order to get the solution at each interior point at the selected domain where problems is defined. After discretization is incorporated we come across a difference equation. A suitable process must be selected with designed algorithm to reach numerical solution. Numerically computed solution must be analyzed with the analytical solution for constancy, stability and convergence. The selected problems are available in $[1,3,12]$ where numerical solutions are discussed and developed the consistency, stability and convergent analysis mechanism are discussed. In [6,9] we can see the various methods to solve differential equations computationally. In the literature study $[4,5,7,12]$ various numerical methods are developed and discussed. We have attempted to solve a two-point BVP using CS approximation method and three-term recursive algorithm [14, 15]. Since closed form solutions for most of these problems are not available so one has to resort to get the solutions of such problems in approximation technology. Due to the advent of digital computer it has become possible to consider and solve such a huge task. The most commonly employed approximate methods for solving such problems are Finite Difference Methods, Numerical Quadrature method and Finite Volume Methods. In this present research paper a two point BVP with Neumann BCs are considered. Taylor's series can be used as an approximation to convert a second order DE into a first order 
DE. Such first order differential equation again transformed into a finite difference equation. Numerical integration method subsequently Thomas algorithm employed to get the approximate solution in the defined region. It is observed that numerically computed solutions ae in good agreement on par with the available closed form solutions.

Two point BVPs occur very frequently in branches of Engineering. Since closed form solutions are not possible for all we try to find solutions of such problems using approximations. The most frequently adoptable approximate methods for solving such problems are FDM, Numerical quadrature method and FVM.

In this present research problem a two point BVP with differential BCs are considered. Taylor's series approximation is used to reduce a second order DE into first order differential equation subsequently the first order DE transformed into a finite difference equation. Numerical integration method and subsequently Thomas algorithm employed to get the approximate solution in the defined region at each mesh point for the various perturbation parameter values. It is observed that numerically computed solutions are in line with available closed form solutions.

\section{EXPLANATION}

Two-point BVPs with singular pertubation is given by

$$
\varepsilon y^{\prime \prime}(x)+\frac{c}{x} y^{\prime}(x)+b(x) y(x)=d(x), \quad 0<x<1
$$

Where $\varepsilon$ is a pertubed parameter very close to zero such that $0<\varepsilon \leqslant<1$ and $c \geq 1$ with BCs

$$
y^{\prime}(0)=\alpha, \quad y(1)=\beta
$$

We try to modify (1) at $x=0$ (singular point) to get

$$
\begin{aligned}
& y^{\prime \prime}(x)+p(x) y^{\prime}(x)+q(x) y(x)=r(x), \\
& y^{\prime}(0)=\alpha, \quad y(1)=\beta
\end{aligned}
$$

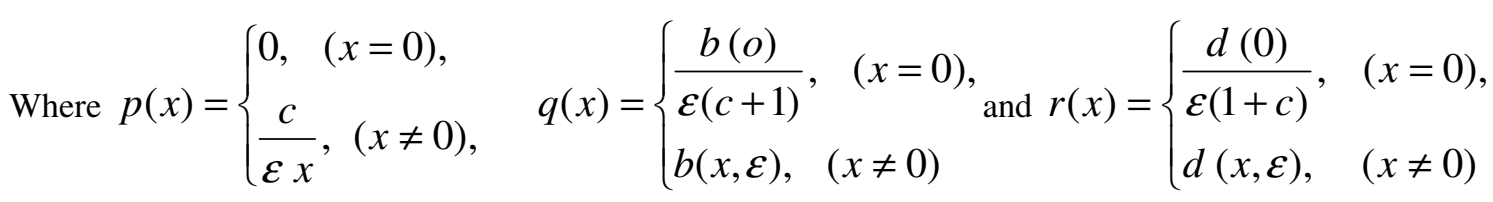

Here assume the grid points of the mesh as $x_{i}$ with $x_{0}=0, x_{n}=1$ with $h=x_{i}-x_{i-1}>0$ and construct the spline function as given below.

\section{Construction of Spline Function}

For the given set of data points $\left(\mathrm{x}_{\mathrm{i}}, \mathrm{y}_{\mathrm{i}}\right)$ the data defined $(\mathrm{i}=0,1,2,3,4 \ldots \mathrm{n})$ and let $\mathrm{S}_{\mathrm{i}}(\mathrm{x})$ be the CS defined in the interval [ $\left.x_{i,} x_{i+1}\right]$. As we aware of the conditions of it are

- $\quad \mathrm{S}_{\mathrm{i}}(\mathrm{x})$ is approximately cubic in every sub-interval $\left[x_{i}, x_{i+1}\right], \mathrm{i}=0,1,2,3, \ldots \ldots, \mathrm{n}-1$

- $\quad \mathrm{S}_{\mathrm{i}}(\mathrm{x})=\mathrm{y}_{\mathrm{i}}$

- $\quad \mathrm{S}_{\mathrm{i}}(\mathrm{x}), S_{i}^{l}(x)$ and $S_{i}^{\prime \prime}(\mathrm{x})$ are continuous in $\left[x_{0}, x_{n}\right]$ 
- $S_{i}^{\prime \prime}\left(x_{0}\right) S_{i}^{\prime \prime}\left(x_{n)}=0\right.$

For deriving the associated equations of the cubic spline, we perceive the second derivatives of spline to be of first

degree. So we consider the condition in $\mathrm{S}_{\mathrm{i}}(\mathrm{x})$

$$
S_{i}^{\prime \prime}(x)=\frac{1}{h_{i}}\left[\left(x_{i}-x\right) M_{i-1}+\left(x-x_{i-1}\right) M_{i}\right]
$$

Where $h$ is the step width defined by

$\mathrm{h}=x_{i}-x_{i-1} \quad, \quad S_{i}^{\prime \prime}\left(x_{i}\right)=M_{i}$ for all $\mathrm{i}$. It is evident that the spline or smooth curves that connect two are more specific points.

Integrating (5) two times with respect to the independent variable we have

$$
S_{i}(x)=\frac{1}{h_{i}}\left[\left(x_{i}-x\right)^{3} M_{i-1}+\frac{\left(x-x_{i-1}\right)^{3}}{6} M_{i}\right]+c_{i}\left(x_{i}-x\right)+d_{i}\left(x-x_{i-1}\right)
$$

Where $c_{i} \& d_{i}$ are constants to be calculated.

Apply the conditions $s_{i}\left(x_{i}\right)=y_{i} a n d s_{i}\left(x_{i+1}\right)=y_{i+1}$ so we have

$$
c_{i}=\frac{1}{h_{i}}\left[y_{i-1}-\frac{h_{i}^{2}}{6} M_{i-1}\right], d_{i}=\frac{1}{h_{i}}\left[y_{i}-\frac{h_{i}^{2}}{6} M_{i}\right]
$$

Substitute $c_{i} \& d_{i}$ in equation (6) we have

$$
S_{i}(x)=\frac{1}{h_{i}}\left[\begin{array}{l}
\frac{\left(x_{i}-x\right)^{3}}{6} M_{i-1}+\frac{\left(x-x_{i-1}\right)^{3}}{6} M_{i}+ \\
\left(y_{i-1}-\frac{h_{i}^{2}}{6} M_{i-1}\right)\left(x_{i}-x\right)+ \\
\left(y_{i}-\frac{h_{i}^{2}}{6} M_{i}\right)\left(x-x_{i-1}\right)
\end{array}\right]
$$

In (8) the spline second derivatives $M_{i}$ are not known still. To calculate them we apply the other condition of continuity of $S_{i}^{r}(x)$. Differentiate Equation (8) one can have

$$
S_{i}^{\prime}(x)=\frac{1}{h_{i}}\left[\begin{array}{l}
-3\left(\frac{\left(x_{i}-x\right)^{2}}{6}\right) M_{i-1}+\frac{3\left(x-x_{i-1}\right)^{2}}{6} M_{i} \\
-\left(y_{i-1}-\frac{h_{i}^{2}}{6} M_{i-1}\right)+y_{i}-\frac{h_{i}^{2}}{6} M_{i}
\end{array}\right]
$$

Since $\mathrm{S}_{\mathrm{i}}(\mathrm{x})$ is differentiable function so it's right hand and left hand derivatives exists and they are evidently equal and for equal intervals $h_{i+1}=h_{i}=h$ 


$$
\begin{aligned}
S^{\prime}(x)= & M_{i-1}\left[\frac{h}{6}-\frac{\left(x_{i}-x\right)^{2}}{2 h}\right]+M_{i}\left[\frac{\left(x-x_{i-1}\right)^{2}}{2 h}-\frac{h}{6}\right] \\
& +\frac{\left(y_{i}-y_{i-1}\right)}{h}
\end{aligned}
$$

From (10), we get

$$
\begin{aligned}
& S^{\prime}\left(x_{i}^{+}\right)=-\frac{h}{3} M_{i}-\frac{h}{6} M_{i+1}+\frac{y_{i+1}-y_{i}}{h}, \quad i=0,1,2 \ldots \ldots ., n-1 \\
& S^{\prime}\left(x_{i}^{-}\right)=\frac{h}{3} M_{i}+\frac{h}{6} M_{i-1}+\frac{y_{i}-y_{i-1}}{h}, \quad i=0,1,2 \ldots \ldots ., n
\end{aligned}
$$

The continuity condition $S^{\prime}\left(x_{i}^{+}\right)=S^{\prime}\left(x_{i}^{-}\right)$gives

$$
\frac{h}{6} M_{i-1}+\frac{2 h}{3} M_{i}+\frac{h}{6} M_{i+1}=\frac{y_{i+1}-2 y_{i}+y_{i-1}}{h}, \quad i=1,2 \ldots \ldots ., n-1
$$

By using it in any sub-interval $\left[x_{i}, x_{i+1}\right]$ with $h_{i+1}=x_{i+1}-x_{i}$

For equal intervals $h_{i+1}=h_{i}=h$ we can write by utilizing eqn. (8) the following

$$
\begin{aligned}
& M_{i-1}+4 M_{i}+M_{i+1}=\frac{6}{h^{2}}\left(y_{i+1}-2 y_{i}+y_{i-1}\right), \\
& \mathrm{i}=1,2, \ldots \ldots ., \mathrm{n}-1
\end{aligned}
$$

The simultaneous system of equations (14) has special significance. If $M_{0}$ and $M_{n}$ are known, then equation (14) can be transformed to

$$
\begin{aligned}
& h_{n-1} M_{n-2}+2\left(h_{n-1}+h_{n}\right) M_{n-1}= \\
& 6\left[\frac{y_{n}-y_{n-1}}{h_{n}}-\frac{y_{n-1}-y_{n-n}}{h_{n-1}}\right]-h_{n} M_{n}
\end{aligned}
$$

Equation (14)is an (n-1) system of equations along with two conditions in (4) for natural spline, the complete system can be solved for the values $M_{i}$ ( $\left.\mathrm{i}=1,2 \ldots . \mathrm{n}-1\right)$. The system of equations (15) as tri-diagonal which can be solved by Gaussian elimination method or by applying efficient and accurate method known as Thomas algorithm (simple version of Gauss elimination) when the $M_{i}$ are known. So that equation (8) gives the required cubic spline in each sub-interval $\left[x_{i-1}, x_{i}\right]$. Also the numerical derivatives can be calculated from using (9) with the virtue of finite slopes in each subinterval.

$$
\begin{aligned}
& S(x)=M_{i-1} \frac{\left(x_{i}-x\right)^{3}}{6 h}+M_{i} \frac{\left(x-x_{i-1}\right)^{3}}{6 h}+ \\
& \left(y_{i-1}-\frac{h^{2}}{6} M_{i-1}\right)\left(\frac{x_{i}-x}{h}\right)+\left(y_{i}-\frac{h^{2}}{6} M_{i}\right) \frac{\left(x-x_{i-1}\right)}{h}
\end{aligned}
$$


Where $M_{i}=S^{\prime \prime}\left(x_{i}\right)$ and $y_{i}=y\left(x_{i}\right)$. If the function $y(x)$ at all points $y(x) \varepsilon C^{4}[0,1]$, then the CS function $S(x)$ approximates $y(x)$ at all points in the interval [0,1] up to fourth order. The unknowns $\mathrm{M}_{\mathrm{i}}$ are connected by continuity condition on $S^{\prime}(x)$.Use Eq. (2) at equidistant grid points $x_{i}=x_{0}+i h, \mathrm{i}=0,1,2, \ldots . \mathrm{n}$ to determine Cubic Spline interpolating polynomial. Using a known approach [2] , we have

$$
A_{i} y_{i-1}-B_{i} y_{i}+C_{i} y_{i+1}=D_{i}, i=1,2, \ldots \ldots, n-1
$$

Where

$$
\begin{aligned}
& A_{i}=b_{i}\left[1-\frac{h}{2} p_{i-1}+\frac{h^{2}}{6} q_{i-1}\right], B_{i}=a_{i}\left[1+\frac{h}{2} p_{i+1}\right]+b_{i}\left[1-\frac{h}{2} p_{i-1}\right]-\frac{2 h^{2}}{3} q_{i} d_{i}, \\
& C_{i}=a_{i}\left[1+\frac{h}{2} p_{i+1}+\frac{h^{2}}{6} q_{i+1}\right], D_{i}=\frac{h^{2}}{6}\left(b_{i} r_{i-1}+4 e_{i} r_{i}+a_{i} r_{i+1}\right), \\
& a_{i}=1-\frac{h}{3} p_{i-1}+\frac{h}{3} p_{i}-\frac{h^{2}}{12} p_{i} p_{i-1}, b_{i}=1-\frac{h}{3} p_{i}+\frac{h}{3} p_{i+1}-, \frac{h^{2}}{12} p_{i} p_{i+1}, \\
& e_{i}=1-\frac{h^{2}}{12} p_{i-1} p_{i+1}+\frac{7 h}{24}\left(p_{i+1}-p_{i-1}\right) .
\end{aligned}
$$

The BCs (3) and (4) in discrete form is given by

$$
\begin{aligned}
& S_{0} y_{0}=\alpha S_{1} y_{1}+S_{2}, \\
& y_{n}=\beta \\
& S_{0}=\frac{h}{3 a_{1}}\left(q_{0}-\frac{p_{0}}{h}-\frac{p_{1}}{2 h}-\frac{p_{0} p_{1}}{4}+\frac{h}{4} p_{1} q_{0}-\frac{3 a_{1}}{h^{2}}\right) \\
& S_{1}=\frac{h}{3 a_{1}}\left(-\frac{p_{0}}{h}-\frac{q_{1}}{2}-\frac{p_{1}}{2 h}-\frac{p_{0} p_{1}}{4}-\frac{3 a_{1}}{h^{2}}\right), \\
& S_{2}=\frac{h}{3 a_{1}}\left(r_{0}+\frac{r_{1}}{2}+\frac{h}{4} p_{1} r_{0}\right),
\end{aligned}
$$

Eq. (17) together with Eqs.(18) and (19) form ' $n+1$ 'system of equations with $n+1$ unknowns which would be used to solve the unknowns. This System has been solved using the simple algorithm defined by Thomas. The algorithm pivots the unknown values by back substitution method.The starting recurrence relation we use is

$$
y_{i}=W_{i} y_{i+1}+T_{i}, i=0,1,2, \ldots \ldots . n-1
$$

Make Use of Eqn (23) in (17) to get 


$$
\begin{aligned}
& W_{i}=\frac{G_{i}}{F_{i}-E_{i} W_{i-1}} \\
& \text { and } T_{i}=\frac{E_{i} T_{i-1}-H_{i}}{F_{i}-E_{i} W_{i-1}}
\end{aligned}
$$

The value of $W_{0}$ and $T_{0}$ and required to find $W_{i}$ and $T_{i}$

From (18), we have

$$
\begin{gathered}
W_{0}=\frac{S_{1}}{S_{0}}, \\
T_{0}=\frac{S_{2}}{S_{0}}
\end{gathered}
$$

We compute $W_{i}$ and $T_{i}$ from Eqn (24) and (25) using forward process with the initial conditions .

Example Problems: To test the efficianccy of the selected method we have given some exampels into consideration. The patterns revealed the tendency of the solution.

\section{Numerical Examples}

\section{Example-1}

We take a second order DE with Neumann boudary conditons

$$
\begin{aligned}
& -y^{\prime \prime}(x)-\frac{2}{x} y^{\prime}(x)+\left(1-x^{2}\right) y(x)=x^{4}-2 x^{2}+7, \\
& y^{\prime}(0)=0, \quad y(1)=0
\end{aligned}
$$

The exact solution is $y(x)=1-x^{2}$ and numerical approximations are given below.

Table 1: Numerical Results for Example 1

\begin{tabular}{|c|c|c|c|}
\hline Argument(x) & $\begin{array}{c}\text { Step } \\
\text { width(h=0.05) }\end{array}$ & $\begin{array}{c}\text { Step width } \\
\mathbf{h = 0 . 0 2 5}\end{array}$ & $\begin{array}{c}\text { Exact } \\
\text { solution }\end{array}$ \\
\hline 0 & 0.99998 & 1.000002 & 1.000000 \\
\hline 0.025 & 0.999370 & 0.999377 & 0.999375 \\
\hline 0.050 & 0.997490 & 0.997502 & 0.995700 \\
\hline 0.1 & 0.989995 & 0.990002 & 0.990000 \\
\hline 0.2 & 0.959998 & 0.960002 & 0.960000 \\
\hline 0.3 & 0.909997 & 0.910001 & 0.910000 \\
\hline 0.4 & 0.839988 & 0.840001 & 0.840000 \\
\hline 0.5 & 0.749999 & 0.750001 & 0.750000 \\
\hline 0.8 & 0.359998 & 0.359884 & 0.360000 \\
\hline 0.9 & 0.190002 & 0.190000 & 0.190000 \\
\hline 1 & 0.000000 & 0.000000 & 0.000000 \\
\hline
\end{tabular}




\section{Example-2}

Consider the following BVP

$$
\frac{d^{2} y}{d x^{2}}+\frac{2}{x} \frac{d y}{d x}-4 y=-2
$$

with the prescribed Neumann BCs

$\frac{d y}{d x}=0$ at $\mathrm{x}=0, \mathrm{y}=5.5$ at $\mathrm{x}=1$. The analytical solution for example- 2 is given by

$$
y(x)=\frac{1}{2}+\frac{5 \sinh 2 x}{x \sinh 2}
$$

The values have been compared and tabulated in Table 2

Table 2: Numerical Results for Example 2

\begin{tabular}{|c|c|c|c|}
\hline $\begin{array}{c}\text { Argument } \\
\text { value (x) }\end{array}$ & $\begin{array}{c}\text { Spline method } \\
(\mathbf{h}=\mathbf{0 . 0 5})\end{array}$ & $\begin{array}{c}\text { Spline } \\
\text { method(h=0.025) }\end{array}$ & $\begin{array}{c}\text { Analytical } \\
\text { solution }\end{array}$ \\
\hline 0 & 3.256907 & 3.256904 & 3.257206 \\
\hline 0.1 & 3.275328 & 3.275299 & 3.275624 \\
\hline $0-2$ & 3.331029 & 3.331006 & 3.333132 \\
\hline 0.3 & 3.425356 & 3.425289 & 3.425641 \\
\hline 0.4 & 3.560588 & 3.560566 & 3.560864 \\
\hline 0.5 & 3.740011 & 3.740009 & 3.740271 \\
\hline 0.6 & 3.977901 & 3.977851 & 3.968246 \\
\hline 0.7 & 4.240188 & 4.240169 & 4.250393 \\
\hline 0.8 & 4.592999 & 4.592878 & 4.593706 \\
\hline 0.9 & 5.000623 & 5.000586 & 5.006766 \\
\hline 1 & 5.599990 & 5.599658 & 5.500000 \\
\hline
\end{tabular}

Similary we can generate numerical approximation values by lowering the step width accordingly we can get the most plausible values similar to the above tables.

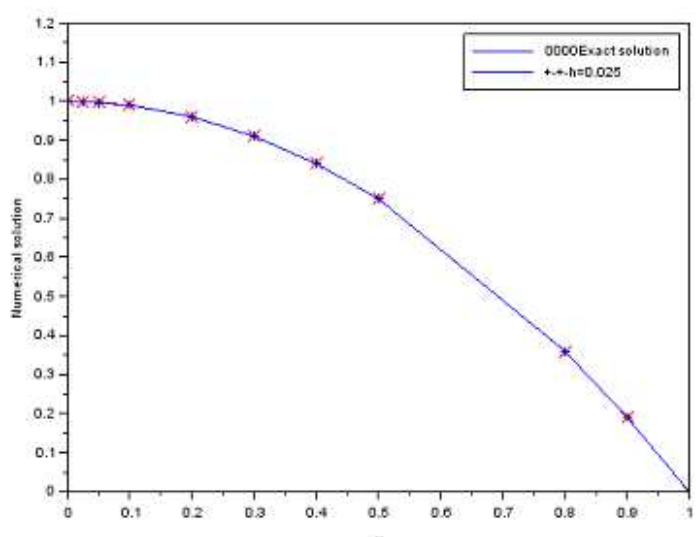

Figure 1 


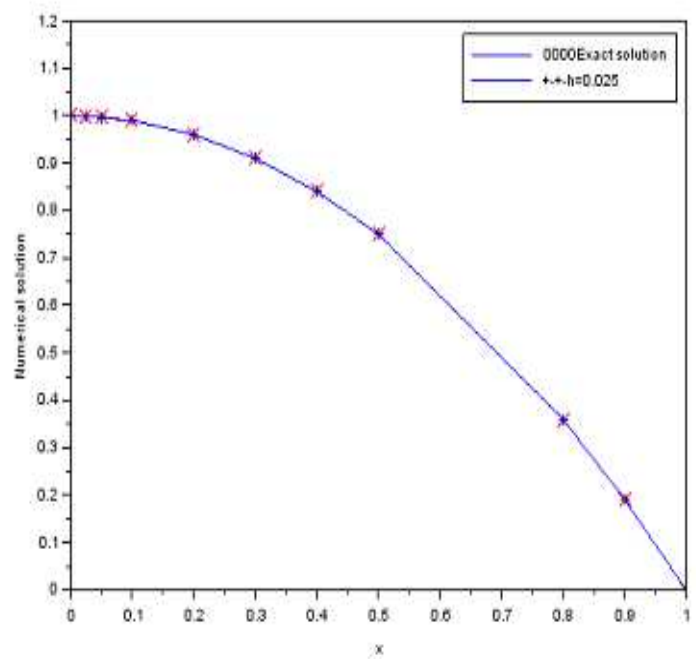

Figure 2

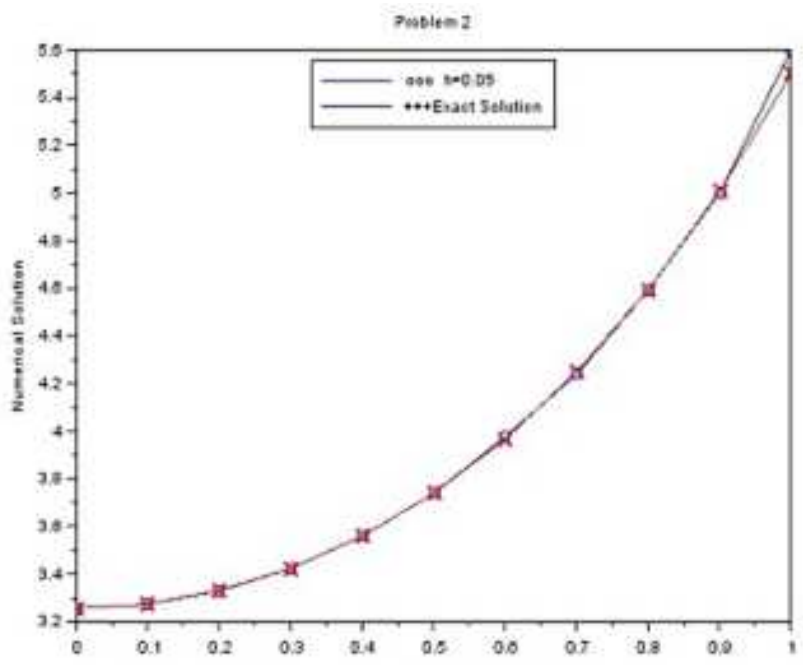

Figure 3

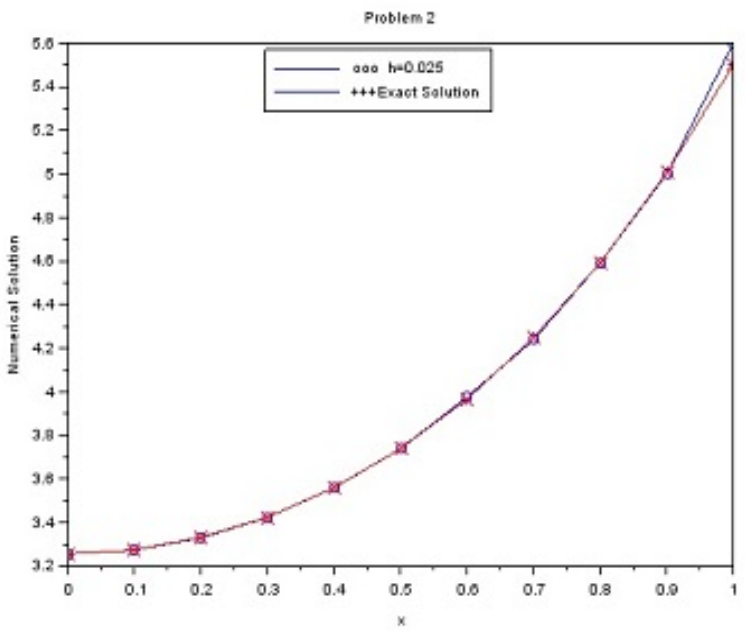

Figure 4 


\section{CONCLUSIONS}

We have defined a two point BVP with prescribed BCs, described and demonstrated the methodology of the applied CS method for solving BVPs with single perturbation. We have analysed, developed and designed a descrete three term recursive relation by using this method. Such three term recursive difference equation is solved using Thomas algorithm. This method is simple, precise, elegant and user friendly to be implemented on a computer. However here we have implemented this method with some selected problems available in the literature due to comarative study. More particularly examples of homogeneous and non -homogeneous singular BVPs are considered and implemented. Numerically obtained results are tabulated and complared with the exact solutions or analytical solution. After comparative study we come to a conclution that the numerical results are in perfect correlation with exact solutions available in the literatue. The algorithm has a strong convergent analogy by obesrving the tabulated values throughout the region. Even for small step size also the tendency of the convergence is retained throughout the domain. We establish here that the method used here is in correlation with exact solution to a great extent. The convergence, consistancy and stability of the solution is uniform throughout the defined region.

\section{REFERENCES}

1. Caglar, H., Caglar, N., Elfaituri, K., B-Spline Interpolation Compared with Finite difference, Finite Element and Finite Volume Methods Which Applied to Two-Point Boundary Value Problems, Applied Mathematics and Computation, (175), 2006, pp. 72-79.

2. C.G.Lange, R. M.Miura, Singular Perturbation Analysis of Boundary-Value Problems for Di $\square$ erential-Di $\square$ erence Equations. v.small shifts with layer behavior, SIAM J. Appl. Math. 54(1994), 249-272.

3. Fang, Q., Tsuchiya, T., Yamamoto, T., Finite Difference, Finite Element and Finite Volume Methods Applied to Two-Point Boundary Value Problems, Journal of Computational and Applied Mathematics, (139), 2002, pp. 9-19.

4. Finlayson.B.A, The method of weighted residual and variational Principles, Academic Press,

5. Henrici, P., Discrete Variable Methods in Ordinary Differential Equations, Wiley, New York, 1962.

6. Jain, M.K., Numerical Solution of Differential Equations, Second edition, Wiley Eastern, New Delhi, 1984.

7. Viswanadham, KNS Kasi, and S. M. Reddy. "Numerical solution of fourth order boundary value problems by Petrov-Galerkin Method with cubic B-splines as basis functions and Quintic B-Splines as weight functions." International Journal of Computer Applications 975: 8887.

8. J.Mohapatra, S.Nateshan, Uniformly Convergent Numerical Method for Singularly Perturbed Di $\square$ erential-Di $\square$ erence Equation Using Grid Equi-distribution, Int. J. Numer. Methods Biomed. Eng. 27(2011), 1427-1445.

9. L.E. El'sgolt's, S. B.Norkin, Introduction to the Theory and Application of Differential Equations with Deviating Arguments, Academic Press, New York - London, 1973.

10. Sushanth, Kallem, Raj Kumar, and Anil Bhardwaj. "Soil mapping of Patiala-Ki-Rao watershed in Shivalik Foot-Hills using GIS." International J. of Agri. Sci. Res.(IJASR) 9.2 (2019): 1-8.

11. N. Srinivasacharyulu, K. Sharathbabu (2008), 'Computational method to solve Steady-state Convection-diffusion problem, International Journal of Mathematics, Computer Sciences and Information Technology, Vol. 1 No. 1-2,January-December 2008,pp.245-254.

12. P.B. Patil., U.P Verma., Numerical Computational Methods, Narosa publishing, House, 2006. 
13. Rashidinia, J., Applications of Splines to the Numerical Solution of Differential Equations, Ph.D. Thesis, Aligarh Muslim University, Aligarh, India, 1994.

14. R.S.Varga, Matrix Iterative Analysis, Prentice-Hall, New Jersey, 1962.

15. S.V. Patankar. Numerical Heat Transfer and Fluid Flow. Hemisphere Publishing Corp., Taylor \&Francis Group, New York, 1980.

16. [Numerical solution of Differential equations by M.K.Jain, New Age International publications.

17. Sonawane, Pramod R., and K. B. Chaudhari."Reversible image watermarking using adaptive prediction error expansion and pixel selection." International Journal Of Engineering Science And Innovative Technology (Ijesit) 2.2 (2013).

18. Introductory methods of numerical Analysis by S.S. Sastry, Prentice Hall publications. +

19. Ch.Achi Reddy and B.Shankar (2017) "Soret and Dufour Effects on unsteady MHD boundary Layer flow of a Nano fluid due to an exponentially stretching sheet” Journal of Nano fluids, Vol. 6, PP. 1-9.

20. Reddy, G. Sreeram, and Manzoor Hussain. "A Novel Approach to Compute Using Reverse Eng."International Journal of Mechanical and Production Engineering Research and Development (IJMPERD) 6.5, Oct 2016, 17-24 